\title{
Research on the variability of the burnishing force during processing surfaces with 3D shape by using simultaneous 5-axis ball-burnishing process implemented on CNC milling machine
}

\author{
Stoyan Dimitrov Slavov ${ }^{1}$ and Iliyan Velichkov Iliev ${ }^{1}$ \\ 1 - Technical University of Varna, Department of Mechanical Engineering and Machine Tools, 9010, 1 Studentska Street, Varna, Bulgaria \\ Corresponding author contact: sdslavovetu-varna.bg
}

\begin{abstract}
The present research is focused on continuously measuring the variability of the burnishing force during conducting ball-burnishing process for specimens with surfaces with $3 D$ shape, by using CNC milling machine with dual rotary table installed. For measuring the burnishing force and its variability, the specifically developed ball-burnishing tool with miniature force sensor was used. To assess the degree of influence of the main regime parameters on the variability of the burnishing force, the four factors full factorial experiment design with two levels per factor and four replications per run has been carried out. The experimental results are processed statistically and techniques such as Pareto and ANOVA were used after that, for sorting them by degree of significance. Some conclusions about the magnitude and the causes of the obtained variability also are given.
\end{abstract}

Keywords: ball-burnishing process, pressing force, 5-axis CNC milling machine, full factorial experiment design, Pareto chart analysis, analysis of variance, DOE

\section{$1 \quad$ Introduction}

It is well known, that implementation of ball-burnishing (BB) or vibratory ball-burnishing (VBB) processes as finishing operations in the processing of machine parts with different macro-shape, leads to some operational improvements such as better surface roughness, increase of the fatigue strength, corrosion and wear resistance, etc. There are many examples from conducted experimental studies (Akkurt et all, 2014; Sagbas, 2011; Odintsov, 1987; López de Lacalle et all, 2011; Gharbi et all, 2015; Grochała et all, 2014; Prevéy et all, 2004, 2005; Schneider, 2001), which confirms the positive impact of these processes on operational parameters of parts with different functions and applications. Many of them are related to study the effects of the regime parameters of the BB processes on the surface quality and/or physical and mechanical characteristics of the burnished surfaces. However, most of them were carried out using work-pieces, which have relatively simple macro-shape of the burnished surfaces (for example cylindrical or planar macro-shape). The literature sources (Prevéy et all, 2004, 2005; Schneider, 2001) referred to some schemes for BB or VBB processes of surfaces with more complex macro form, such as hemispherical and/or elliptical reflectors (from lighting or heating units), ship propeller blades, turbine blades, etc., in order to obtain a specific roughness and improve their operational properties. It usually requires the ball tool to perform complex spatial motions in three-dimensional space, following macro-curvature of the work-piece surface. Therefore, CNC milling machines that can perform multiaxis simultaneous milling operations are often used for their realization. This is necessary because in order to obtain homogeneous qualitative, physical, and mechanical parameters overall complex surface processed by $\mathrm{BB}$ or VBB, it is not enough the ball-tool only to travel equidistantly, but its axis of symmetry must always be perpendicular to the momentary curvature of the burnished surface (Apro, 2009). For example in Fig. 1, a) is shown a simplified diagram for BB process of two kinds of surfaces: pos. 1 - planar (2D) and pos. 2 - three-dimensional (3D) surface. In the case of a 2D surface, the reaction vector $\mathrm{R} 1$, [N] (assuming ideal contact conditions) is directed perpendicular to the opposite direction of the burnishing force $\mathrm{F},[\mathrm{N}]$ (see pos. 3), and this position is kept constant during the operation. When the burnished surface has 3D macro-shape, the reaction vector $\mathrm{R} 2,[\mathrm{~N}]$ constantly changes its angle and direction, depending on the momentary curvature of the complex surface, even when magnitude of the 
burnishing force $\mathrm{F},[\mathrm{N}]$ is constant. This will lead to permanent changes in the regime parameters of burnishing operation, which will inevitably have a negative impact on the quality of surface finish.

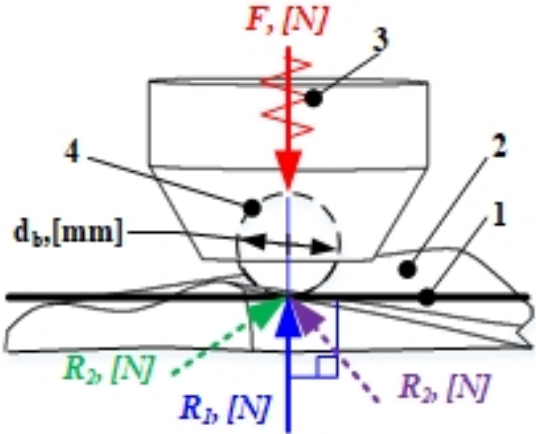

a)

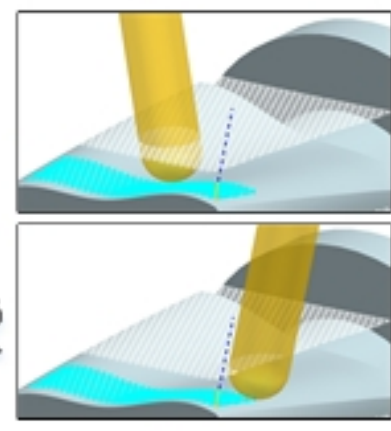

b)

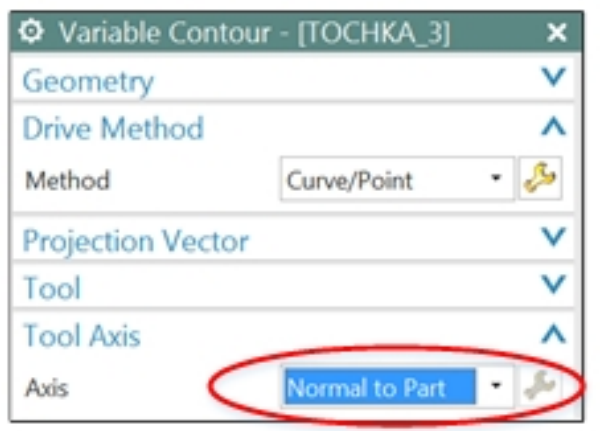

c)

Fig. 1. a) Simplified diagram for BB process: pos. 1 - planar (2D), and pos. 2 - complex 3D surface; b) Modelling BB operation for complex 3D surface by using CAM software; c) Set the option "Normal to part" in the settings of finishing 5-axis milling operations in CAM software.

This problem can be avoided by implementation of the special algorithms for dynamic tilting the tool axis so it is remains always normal to the instantaneous curvature of the ball burnished surface (or to multiple surfaces) of the CAD model. All contemporary CAM software products have built-in such algorithms, and they can be used as additional option in the most 5-axis simultaneous milling operations (Apro, 2009) (see Fig. 1, b, c). However in order to actually perform a BB operation on the real milling machine, the calculated in CAM toolpath must be postprocessed in a suitable NC-code and then red and executed by the CNC system of the machine. Even with today's advances in CAD/CAM capability, due to incorrect choice of suitable postprocessor (or due to unsuitable adjustments in it), and depending on the capabilities and quality of the CNC multi-axis driving system, it is possible certain deviations to be obtained in the real toolpath versus the calculated by CAM optimal (smoothed) trajectory of the ball tool (Apro, 2009). This, along with the dimensional deviations, the clearance in the joints and the elastic deformations of the elements of the technological setup will lead to a certain variability of the burnishing force $\mathrm{F},[\mathrm{N}]$ during the $\mathrm{BB}$ operation. Therefore, it is interesting to research what is actually the variability of the burnishing force during multi-axis simultaneous ball burnishing process, as this may have an impact on the degree of uniformity of resulting plastic deformation in surface layer, and can reduce the effect of the improvements in the surface layer of the burnished workpieces.

In this regard, the main objectives of the present work are to experimentally determine the degree of variability of the compressive force against its nominal value, as well as to identify the main parameters of the regime of the ball-burnishing process that significantly influence this variability..

\section{Methodology of experimental investigation}

\subsection{Setup for experimental measuring the burnishing force during $B B$ process}

In Fig. 2, a, b, are shown the basic components of the experimental setup, used for initially adjustment of the nominal values and for measuring the variability of the burnishing force $\mathrm{F},[\mathrm{N}]$ during $\mathrm{BB}$ processing of complex (3D) surfaces. It is consisting of specially designed deforming ball tool whit integrated miniature force sensor type FCM $\left(\mathrm{F}_{\text {nom }}=5 \mathrm{kN}\right)$ (Slavov \& Iliev, 2016, 2017) (pos. 1), a vertical CNC milling machine HAAS VF3 (pos. 3) with installed dual rotary table type HAAS TR110 (pos. 2) on which are fastened test specimens (pos. 4). The measurement signal from the miniature force sensor enters the conversion device (pos. 5 and 6), which it amplifies and converts from analogue into digital form and then transmitted to the PC (pos. 7), where through specialized software (pos. 8) the data is visualized in digital form and as a graph. The software allows recording the measured data in CSV (comma-separated values) file format, supported by MS Excel for further statistical analysis of the data (Slavov \& Iliev, 2017). 


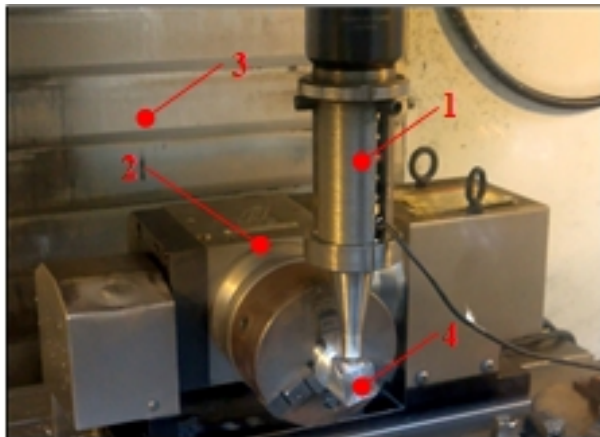

a)

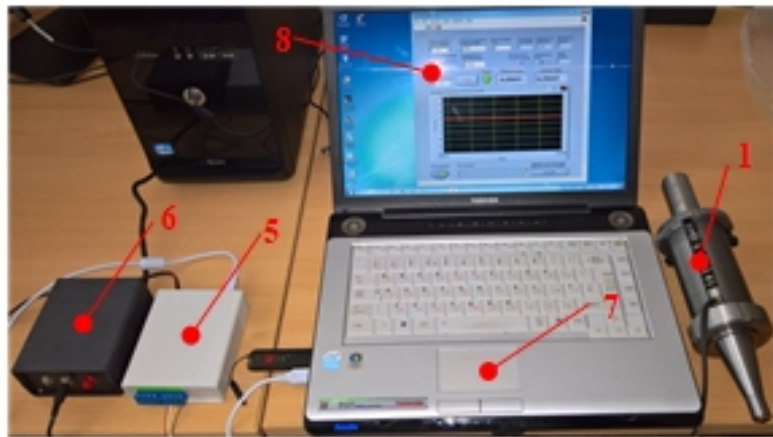

b)

Fig. 2. a) 5-axis setup for BB processing of the 3D surfaces; b) Burnishing force measuring system.

\subsection{Design of the experimental research}

\subsubsection{Description of the experimental specimens}

The experimental specimens are made of aluminum alloy rod, type 2024-T3 with a diameter of $\varnothing 40$, which has physical and mechanical properties, as follows: $\rho=2780 \mathrm{~kg} / \mathrm{m}^{3} ; \mathrm{E}=73 \mathrm{GPa}, \mathrm{Re}=345 \mathrm{MPa}$, $\mathrm{Rm}=483 \mathrm{MPa}, \mathrm{A}=18 \%$, and $\mathrm{HRB}=75$. The shape and dimensions of the specimens, after implementation rough and finish milling operations to obtain needed 3D surfaces for BB process, are shown in Fig. 3, a. The $\mathrm{BB}$ process is carried out as 5-axis simultaneous milling operation with a locked spindle and with enabled mode "Inverse Time" feedrate (G93 code in Fanuc). Every single specimen has four identical surfaces with 3D shaped profile, which corresponding to the four replications of the experimental design plan (see Table 1). Figure 3, b shows example of a specimen, which have specific roughness patterns on the profile surfaces after implementation 5-axis simultaneous burnishing process.

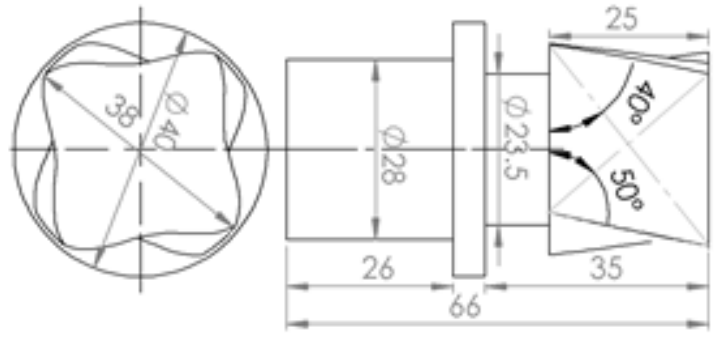

a)

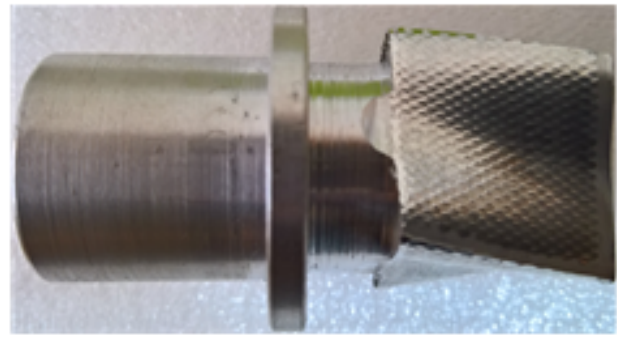

b)

Fig. 3. a) Test specimen with 3D profile surfaces; b) Specific regular shaped roughness on specimen's 3D profile surfaces after implemented BB operation.

\subsubsection{Design of the experimental research.}

To determine the degree of influence of the main regime factors of the BB process, four factors full factorial experiment with two levels per factor of type $2^{4}$ and four replications per run (Slavov \& Iliev, 2017 ) is carried out. The DOE is shown in "Experimental design" section of Table 1.

Based on conducted preliminary studies of the BB process implemented on CNC milling machines (Slavov \& Dimitrov, 2016), the significant (adjustable) regime parameters, which mainly affect the characteristics of the processed surface, have been established. They are as follows:

- $\quad d_{b}, m m$ - diameter of the used ball as burnishing tool (a steel ball from rolling bearing);

- F, N - applied external (burnishing) force by the helical spring of the ball tool;

- $\quad$ e, mm - the set half of the amplitude of the sine wave, related to the width of the obtained cells from roughness with regular microshape;

- $\quad \mathrm{i}$ - the set number of the sinusoids along the path travelled by instrument (Slavov, 2017), which determines the height of the obtained cells from regular roughness; 
Table 1. Full factorial design plan and results for coefficients of variation of the burnishing force F.

\begin{tabular}{|c|c|c|c|c|c|c|c|c|}
\hline \multirow{2}{*}{ Run } & \multicolumn{4}{|c|}{ Experimental design } & \multicolumn{3}{c|}{ Experimental results (coefficients of variation) } \\
\cline { 2 - 9 } & $\mathbf{d}_{\mathbf{b}, \mathbf{~ m m}}$ & $\mathbf{F , ~ N}$ & $\mathbf{e} \mathbf{~ m m}$ & $\mathbf{i}$ & CV (Repl. 1) & CV (Repl. 2) & CV (Repl. 3) & CV (Repl. 4) \\
\hline 1 & 6 & 250 & 0.5 & 1650.15 & 4.351 & 4.307 & 4.263 & 4.229 \\
\hline 2 & 6 & 250 & 0.5 & 2500.15 & 2.550 & 2.867 & 3.046 & 2.809 \\
\hline 3 & 6 & 250 & 1 & 1650.15 & 2.848 & 2.858 & 3.149 & 3.078 \\
\hline 4 & 6 & 250 & 1 & 2500.15 & 3.512 & 3.635 & 3.719 & 3.721 \\
\hline 5 & 6 & 380 & 0.5 & 1650.15 & 0.178 & 0.177 & 0.179 & 0.172 \\
\hline 6 & 6 & 380 & 0.5 & 2500.15 & 0.362 & 0.357 & 0.355 & 0.353 \\
\hline 7 & 6 & 380 & 1 & 1650.15 & 0.324 & 0.533 & 0.327 & 0.321 \\
\hline 8 & 6 & 380 & 1 & 2500.15 & 0.461 & 0.461 & 0.441 & 0.422 \\
\hline 9 & 8 & 250 & 0.5 & 1650.15 & 4.086 & 4.074 & 4.095 & 4.274 \\
\hline 10 & 8 & 250 & 0.5 & 2500.15 & 3.946 & 3.982 & 3.972 & 4.154 \\
\hline 11 & 8 & 250 & 1 & 1650.15 & 4.385 & 4.455 & 4.398 & 4.441 \\
\hline 12 & 8 & 250 & 1 & 2500.15 & 4.875 & 4.928 & 4.919 & 4.897 \\
\hline 13 & 8 & 380 & 0.5 & 1650.15 & 1.148 & 1.138 & 1.100 & 1.035 \\
\hline 14 & 8 & 380 & 0.5 & 2500.15 & 0.571 & 0.670 & 0.653 & 0.669 \\
\hline 15 & 8 & 380 & 1 & 1650.15 & 0.508 & 0.550 & 0.597 & 0.574 \\
\hline 16 & 8 & 380 & 1 & 2500.15 & 0.372 & 0.435 & 0.484 & 0.491 \\
\hline
\end{tabular}

For calculation of the slope and the intercept to determine whether the measured data for the burnishing force have significant slope trend a least squares linear regression is used. The magnitudes of the deviations of burnishing force are assessed against the least squares fitted regression line $F(t)=$

$=a \cdot t+b$. Thus, the effect of the irregularities in the geometric shape and dimensions of the experimental specimens is reduced.

\subsubsection{Statistical processing of measured data}

The Descriptive Statistics analysis tool add-in of MS Excel is used for every run and replication of the experimental design in Table 1. Because for different rows of the experimental plan the samples have different sizes and different mean values, the variability of the force is assessed by using the coefficient of variation (CV) (Kutner et all, 2004). The CV is calculated as ratio between the standard deviation $(\sigma)$ and the mean $(\bar{X})$, using the following formula:

$$
C V=(\sigma / \bar{X}) \cdot 100
$$

In the "Experimental results" section of the Table 1 are given calculated CVs by using equation (1). To build the effects plot (see Fig. 4.a), the average effects Avg $g_{\text {low }}$ and Avg high $_{\text {are calculated for the four }}$ regime parameters, using the following equations:

$$
A v g_{\text {low }}=\frac{1}{8} \cdot\left(\sum_{i=1}^{8} C V_{i}^{\text {low }}\right) \quad, \quad A v g_{\text {high }}=\frac{1}{8} \cdot\left(\sum_{i=9}^{16} C V_{i}^{\text {high }}\right)
$$

where: CVilow - are those eight results from Table 1, where the regime parameters have low level values; CVihigh - the other eight results, where the parameters have high level values.

The effect of each of the four regime parameters on the burnishing force variability is calculated using results from the equations (2) and the formula:

$$
E f f_{\left(d_{b}\right),(F),(e),(i)}=A v g_{\text {high }}-A v g_{\text {low }}
$$

For sorting the regime parameters according to their levels of significance (see Fig. 4.b) Pareto analysis technique is used, based on calculated by using equation (3) values of the effects. Additionally an analysis of variance (Kutner et all, 2004) (see Fig. 4.c) is carried out to calculate the Fisher-values of the factors. For confidence level $95 \%$ and total degrees of freedomDF $=($ Runs $\cdot$ Repplications $)-$ $-1=(16 \cdot 4)-1=63$, the critical Fisher value is 3.993 . 


\section{Results and discussion}

Carried out Pareto analysis (see Fig. 3.b) shows that the variation of the burnishing force mainly depends on the magnitude of its nominal value and to a lesser degree from the diameter $\mathrm{d}_{\mathrm{b}}$ of the used ball as tool. With increasing the magnitude of the burnishing force, proportionally decreases observed variability $\Delta \mathrm{F}$ and vice versa, and when increasing the ball diameter the observed variability $\Delta \mathrm{F}$ also increases. The other two regime parameters (i, and e) have a much smaller impact on the variability of the burnishing force. After conducted ANOVA analysis (see Fig. 3.c), the factors $\mathrm{F}$ and $\mathrm{d}_{\mathrm{b}}$ were assessed as significant at the $95 \%$ level of confidence since their Fisher-values $\left(F=1078.746\right.$ and $\left.d_{b}=38.608\right)$ are greater than critical Fisher value $=3.993$. The Fisher-values of the other two regime parameters are less than the determined critical Fisher value. Thus, conducted analysis of variance reinforces the subjective conclusion derived from the Pareto histogram plot.

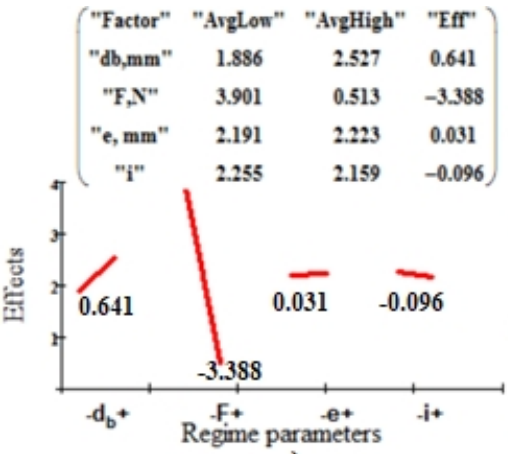

a)

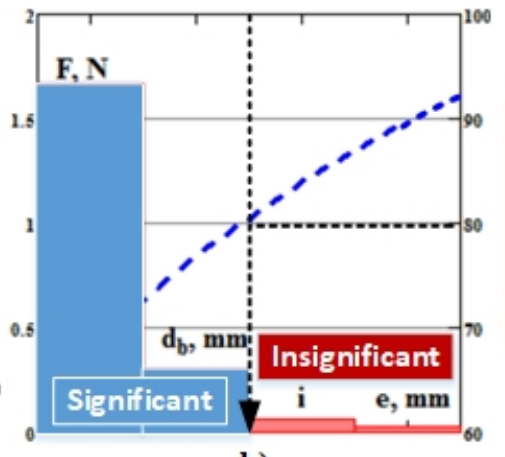

b)

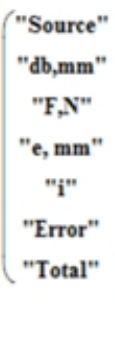

$\left.\begin{array}{ccccc}\text { "SSE" } & \text { "DF" } & \text { "MSE" } & \text { "Fisher" } & \text { "P" } \\ 6.572 & 1 & 6.572 & 38.608 & 0 \\ 183.619 & 1 & 183.619 & 1078.746 & 0 \\ 0.016 & 1 & 0.016 & 0.092 & 0.762 \\ 0.147 & 1 & 0.147 & 0.862 & 0.357 \\ 10.043 & 59 & 0.17 & \mathrm{NaN} & \mathrm{NaN} \\ 200.396 & 63 & \mathrm{NaN} & \mathrm{NaN} & \mathrm{NaN}\end{array}\right)$

c)

Fig. 4. a) Effects plot of the regime parameters; b) Pareto histogram and cumulative graphs, based on the effects; c) ANOVA table with calculated statistics and Fisher-values of the BB process factors.

Obtained results about absolute ranges of the burnishing force variations shows following trends: when the burnishing force has low nominal value $F=250 \mathrm{~N}$, the variability is amended from $\Delta \mathrm{F}_{\min }=37.4$ $\mathrm{N}$ to $\Delta \mathrm{F}_{\max }=53.6 \mathrm{~N}$. At the high level of the force $\mathrm{F}=380 \mathrm{~N}$, this amendment is from $\Delta \mathrm{F}_{\min }=4.5 \mathrm{~N}$ to $\Delta \mathrm{F}=26.9 \mathrm{~N}$. Thus, the range of variability is amended from 15 to $21.5 \%$ at low values and from 1.5 to $7.1 \%$ at high values of the nominally assigned burnishing force. The lower percentage of the obtained force variability is due to a more rigid contact between $3 \mathrm{D}$ profile surfaces of the specimen and the balltool, at the higher values of the nominal burnishing force $\mathrm{F}=380 \mathrm{~N}$, than at lower values $\mathrm{F}=250 \mathrm{~N}$. Increasing the diameter of the ball of the burnished tool from 6 to $8 \mathrm{~mm}$ leads to slightly increase of the observed variability $\Delta \mathrm{F}$. This is mainly due to the lower degree of plastic deformation in the surface layer at the larger diameter of the ball-tool, where the contact conditions are more unstable.

\section{Conclusions}

Based on the conducted experimental research and the undertaken analysis of the obtained results could be made following conclusions:

The experimental setup for 5-axis simultaneous BB process (see Fig. 2 a, b) including multi-axis CNC milling machine with dual rotary table, a ball-tool with miniature force sensor, device for amplification and signal conversion, and specially created software, allows precise measuring the burnishing force during $\mathrm{BB}$ process of 3D profile surfaces from specimens. The specially developed software allows visualization and recording the measurement data in *.CSV file format in real time. This provides reliable subsequent statistical processing of the measurement data in suitable software such as MS Excel, Mathcad, S-Math, etc.

The observed variability of the burnishing force $\Delta \mathrm{F}$ during $\mathrm{BB}$ process carried out by implementing 5 -axis CNC milling machine on 3D profile surfaces mainly depends on the pre-set magnitude of the regime parameter $\mathrm{F}$ and to a lesser extent on the diameter of the ball-tool $\mathrm{d}_{\mathrm{b}}$. The remaining two regimes 
parameters ( $\mathrm{i}$ and e) have a negligible impact on the variability of the burnishing force. This gives grounds they do not take into consideration for future research of the multi-axis BB process.

The measured burnishing force variability reaches a maximum of $7 \%( \pm 3.5 \%)$ at the nominal preset level of the burnishing force $\mathrm{F}=380 \mathrm{~N}$ and $21 \%( \pm 10.5 \%)$, at $\mathrm{F}=250 \mathrm{~N}$. Largely, this mainly due to non-optimal contact between the ball-tool and the 3D surfaces of the specimens at lower values of the nominal burnishing force. The adjusted degree of the accuracy of the postprocessor for generating NCcode for BB process, as well as to smaller stiffness of the used dual rotary table can also be considered as possible reasons for that.

Further investigations have to be done on the influence of postprocessing conditions on the degree of the variability of the burnishing force, especially about the impact of "standard" command G94 (units/time minutes) feedrate and G93 (inverse time) feedrate when the move between the current position in the tool-path and the destination should be completed in 1 divided by the calculated by CAM software feedrate value (Apro, 2009).

\section{References}

A. Akkurt and İ. Ovalı. Improving the surface topography of mild steel with the burnishing process, International Journal of Materials Research, 2014, 105, 10, 1004-1016, DOI: 10.3139/ 146.111112

A. Sagbas, Analysis and optimization of surface roughness in the ball burnishing process using response surface methodology and desirabilty function, Advances in Engineering Software 42 (2011) 992-998.

Gharbi, F., Sghaier, S., Morel, F. et al. J. of Materi Eng and Perform (2015) 24: 721. https://doi.org/10.1007/s11665-014-1349-1

Grochała, D., Berczyński, S. \& Grządziel, Z. Int J Adv Manuf Technol (2014) 72: 1655. https://doi.org/10.1007/s00170-014-5775-X

E. Poursaeidi, M. Salavatian, Failure analysis of generator rotor fan blades, Engineering Failure Analysis 14 (2007) 851-860

K. Apro, Secrets of 5-Axis Machining, Industrial Press Inc., New York, (2009)

L. G. Odintsov, Hardening and Finishing of Parts by Surface Plastic Deformation. Mashinostroenie, Moscow (1987)

Luis N. López de Lacalle, A. Rodríguez, A. Lamikiz, A. Celaya \& R. Alberdi, Materials and Manufacturing Processes, 26/8, 997 (2011) DOI: 10.1080/10426914.2010.529589

Michael H. Kutner, John Neter, Christopher J. Nachtsheim, William Li, Applied Linear Statistical Models (5th ed.), McGraw-Hill Education, (2004)

P. S. Prevéy, N. Jayaraman, J. Cammett, Proc. of ICSP 9 (Paper 260) Paris, (2005)

P. S. Prevéy, N. Jayaraman, R. Ravindranath, Proc. of 9th NTEHCF Conf., USA (2004)

S. D. Slavov, I. Iliev, A study on the variability of the pressing force during processing of regular shaped roughness by using new kinematical scheme for ball-burnishing process, MATEC Web Conf. 11202006 (2017) DOI:10.1051/matecconf/201711202006 
Slavov S., An Algorithm for Generating Optimal Toolpaths for CNC Based Ball-Burnishing Process of Planar Surfaces. In: Proceedings of the Second International Scientific Conference "Intelligent Information Technologies for Industry" (IITI'17), Advances in Intelligent Systems and Computing, vol 680. Springer, Cham, (2017). DOI: 10.1007/978-3-319-68324-9_40

S. Slavov, I. Iliev, Design and FEM static analysis of an instrument for surface plastic deformation of non-planar functional surfaces of machine parts, Fiability\& Durability, ISSN $1844-640$ X, 2, 3-9 (2016)

Yu. G. Schneider, Operational properties of parts with regular microshape, publishing IVA, St. Petersburg, (2001) 